\title{
An Enhanced Anti-Tumor Response by Using Bacterial Magnetosomes Gene Administration Platform
}

\author{
Yishu Tang ${ }^{1 *}$, Chunxia Zhou ${ }^{2}$, Wenbo $\mathrm{Ma}^{2}$, Dongmei Wang ${ }^{2}$, Shuren Zhang ${ }^{2}$ \\ ${ }^{1}$ Department of Laboratory Medicine, The First Affiliated Hospital of Chongqing Medical University, Chongqing, \\ People's Republic of China \\ ${ }^{2}$ Department of Immunology, Cancer Institute, Peking Union Medical College,Chinese Academy of Medical \\ Sciences, Beijing, China
}

*Corresponding Author: Yishu Tang, Department of Laboratory Medicine, The First Affiliated Hospital of Chongqing Medical University, No. 1 Youyi Road, Yuzhong District, Chongqing, 400016, China, Tel: +86 23 89012735; Fax: +86 23 89012735; E-mail: tangyishu111@,163.com

Received: 11 April 2018; Accepted: 16 May 2018; Published: 18 May 2018

\begin{abstract}
DNA vaccination is an effective way to induce specific immunity. In our study, we used magnetosomes (bacterial magnetic particles, BMPs) as the vehicle of a DNA complex of a secondary lymphoid tissue chemokine, human papillomavirus type E7 (HPV-E7) and Ig-Fc fragment (pSLC-E7-Fc) to produce a gene vaccine (BMP-V) for tumor immunotherapy. In a mouse tumour model, intramuscular injection of BMP-V plus magnetic exposure induced E7specific immunity eliciting to tumor inhibition. Taken together, these results demonstrated that BMP could be applied as a DNA vehicle to induce specific immune effect.
\end{abstract}

Keywords: DNA vaccines; Immune effect; Gene delivery; Bacterial magnetic nanoparticles

\section{Introduction}

DNA vaccines were an effective method to generate antigen-specific immunity. Therefore, lots of delivery ways have been studied. The DNA vaccine delivery by conventional intramuscular injection could not induce optimal immunogenicity, even at high concentrations [1]. The gene gun system and electroporation were superior to other methods [2]. However, various factors influence the success of this method, including the electric pulse, buffer, number of pulses, pulse width, cell density and DNA amount. Both systems require specific and expensive instruments [3]. Therefore, an effective and easy gene delivery system is required to solve the present challenges. 
Bacterial magnetic nanoparticles (BMPs), called magnetosomes, were been found in magnetotactic bacteria. BMPs could been used as effective carriers of drugs and genes to target cancer because of their unique properties, such as paramagnetism, nanoscale size $(40-120 \mathrm{~nm})$ and high dispersal quality [4]. BMPs are innovative nanoparticles covered with a stable cytoplasmic membrane, which have presumed good biocompatibility. So BMPs could been used to bind proteins and genes in various application area [5]. The BMP-polyethylenimine (BMP-PEI) gene administration system has been developed, which was lower toxicity and higher transfection efficiency than PEI alone [6].

Our laboratory has developed a chemotactic antigen DNA vaccine system to target the human papillomavirus type16 E7 (HPV-16E7) antigen. The plasmid pSLC-E7-Fc contained a fused gene, in which the E7 gene was sandwiched by the secondary lymphoid tissue chemokine (SLC) and the IgG Fc fragment genes. Our study demonstrated that pSLC-E7-Fc could result in systemic anti-tumour immunity against E7-expressing tumours [7]. In the study, we used the DNA vaccine pSLC-E7-Fc to exemplify the BMPs as a gene carrier. Our results illustrated that BMPs represents a novel and simple approach for gene delivery.

\section{Materials and Methods}

\subsection{Cell lines}

Mouse TC-1 tumour cells, derived from primary epithelial cells of C57BL/6 mice co-transformed with HPV-16 E6 and E7 and c-Ha-Ras oncogene, were a kind gift from Dr TC Wu (Johns Hopkins Medical Institutions, Baltimore, MD, USA) and were cultured in RPMI 1640 medium (Gibco-BRL) supplemented with 10\% fetal calf serum (heatinactivated) and 400mgml G418.

\subsection{Mice}

Inbred female C57BL/6 mice (6-8 weeks of age) were purchased from the Animal Centre of Chinese Academy of Medical Sciences (Beijing, China) and housed under specific pathogen-free conditions.

\subsection{PEI and BMP}

BMPs, which were produced by the bacterium Magnetospirillum gryphiswaldense MSR-1 and PEI (25 kDa from Aldrich, St Louis, MO, USA) were kindly provided by Professor Ying Li (Microbiology Department, College of Biological Sciences, China Agricultural University). All BMPs were sterilised by g-rays (15 kGy).

\subsection{DNA-binding assay}

The polyplexes of DNA, PEI and BMPs were prepared at mass ratios of DNA:PEI:BMP=1:1:0.3. These polyplexes were formed in PBS. The DNA was quantified using spectrophotometry at OD280.

\subsection{Tumour models and treatment protocol}

For the in vivo therapeutic experiment, $5 \times 10^{4} \mathrm{TC}-1$ cells were inoculated s.c. into the right flank of C57BL/6 mice on day 0 . When the tumour reached an average diameter of $5 \mathrm{~mm}$ on day 4 , mice were randomized and treated with 
different doses of BMP-V in $0.1 \mathrm{ml}$ of PBS or PBS alone. Similar treatments were repeated on days 8 and 12. BMP$\mathrm{V}$ was administered to mice intramuscularly to the shaved leg region with or without a magnet (magnetic flux density of $600 \mathrm{mT}$ ) on the injection site for 10min. Tumours were monitored every 3 days by measuring their dimensions using calipers and calculating their volumes according to the following formula: tumour volume $\left(\mathrm{mm}^{3}\right)=0.52\left(\right.$ length $\mathrm{X}$ width $\left.^{2}\right)$.

\subsection{Cytotoxicity assays}

C57BL/6 mice were challenged s.c. with $5 \times 104$ TC-1 cells. Four days later, mice were divided into a control group and an immunisation group, in which they were given PBS or $50 \mu \mathrm{g}$ BMP-V i.m. plus magnet, respectively. Mice were boosted twice on days 8 and 12. Six days after the final booster, splenocytes from immunized and control mice were used as effector cells. B16-F10, TC-1 and YAC-1 cells were used as target cells. Effector cells were added to target cells at a ratio of 60:1,30:1 and 15:1 (tested in triplicate). The supernatants were pooled and measured lactate dehydrogenase using a CytoTox 96 Non-Radioactive Cytotoxicity Assay kit (Promega) according to the manufacturer's instructions.

\subsection{Statistical analysis}

To compare individual time points, analysis of variance was used for comparisons among three or more groups. Student's t-test was used to compare the means between the two groups. Survival data from the animal studies were analyzed using the log-rank test. Differences were considered to be significant when Po0.05. Statistical analysis was performed using commercially available software (SPSS 11.0, SPSS, Chicago, IL, USA).

\section{Result}

3.1 Intramuscular injection with magnet application resulted in an increased tumour inhibition as compared with other methods of BMP-V administration

Mice were challenged with TC-1 tumor cells subcutaneously followed by vaccination with BMP-V, which was administered using various methods. Next, the therapeutic effects of different doses of BMP-V in the TC-1 tumor model were compared. In brief, C57BL/6 mice were vaccinated with $50 \mu \mathrm{g}, 25 \mu \mathrm{g}, 12.5 \mu \mathrm{g}$ BMP-V with or without magnet, $50 \mu \mathrm{g}, 100 \mu \mathrm{g}$ DNA vaccine through intramuscular injection. Figure 1 demonstrated that $50 \mu \mathrm{g}$ BMP-V vaccination by i.m. injection with the magnet systemically inhibited the growth of tumors compared to PBS group $(\mathrm{P}<0.05)$.On the Day 18, the average tumor volume of PBS group was $872.04 \pm 61.73 \mathrm{~mm}^{3}$. The average tumor volume of $50 \mu \mathrm{g}$ DNA vaccine group was $942.68 \pm 79.68 \mathrm{~mm}^{3}$. The average tumor volume of $50 \mu \mathrm{g}$ BMP-V with the magnet group was $390.29 \pm 57.12 \mathrm{~mm}^{3}$.

3.2 Intramuscular injection BMP-V with magnet in established tumors induces innate and adaptive antitumor response

Splenocytes from animals in BMP-V with magnet group demonstrated cytolysis activity toward TC-1 tumor cells in vitro rather than B16-F10 [(Figure 2), 35.20 $\pm 3.96 \%$ vs $5.67 \pm 2.16 \%$ at E:T=60:1], whereas splenocytes from PBS treated mice failed to demonstrate lysis toward either TC-1 or B16-F10. Thus, it appears that a measurable CTL 


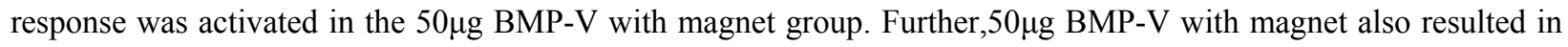
significant cytotoxicity against NK-sensitive YAC-1 cells.

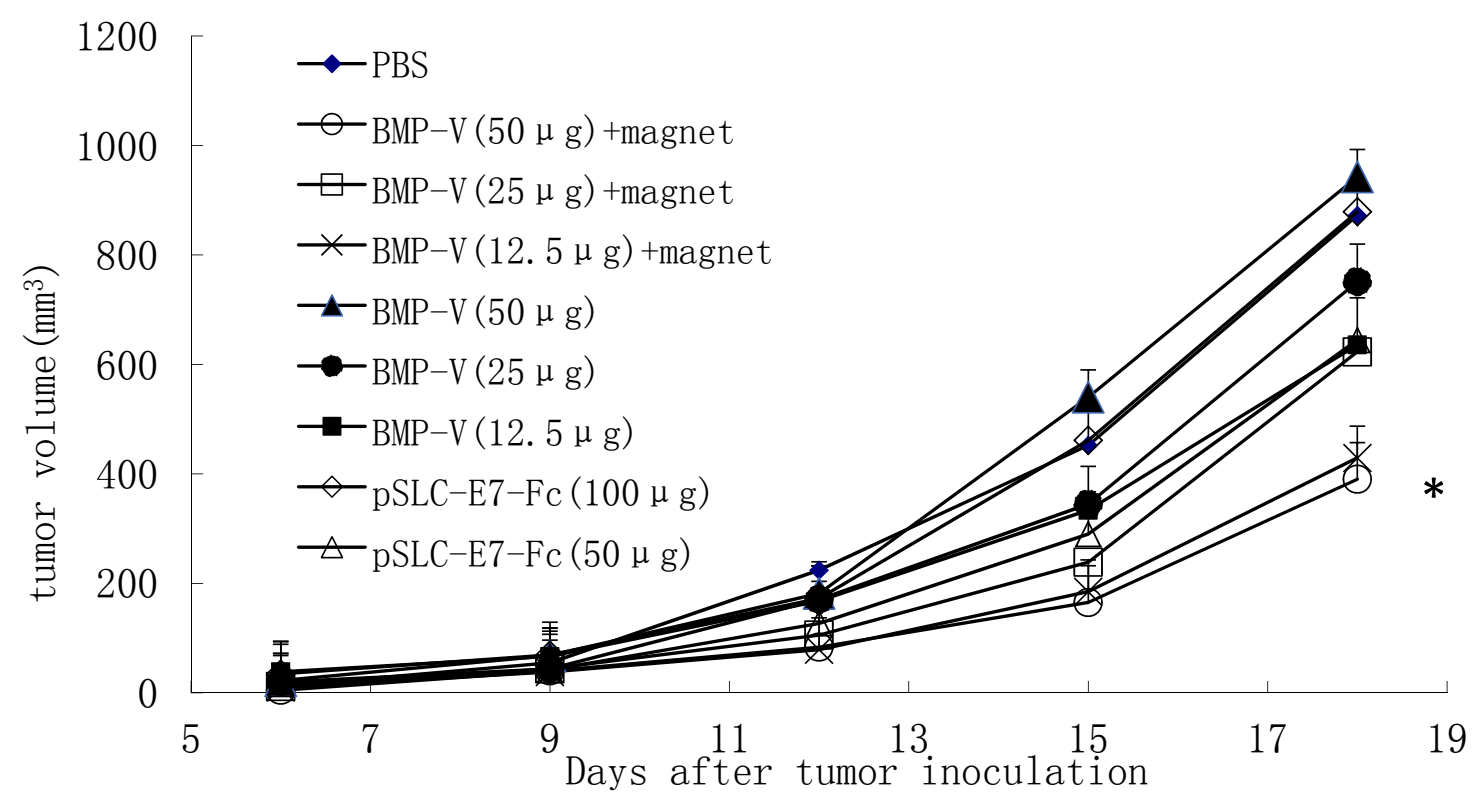

Figure 1: C57BL/6 mice inoculated s.c. with $5 \times 10^{4} \mathrm{TC}-1$ cells. When tumours reached an average diameter of $5 \mathrm{~mm}$ on day 4 , mice were randomised and treated with different doses of BMP-V (50 $\mu \mathrm{g}, 25 \mu \mathrm{g}, 12.5 \mu \mathrm{g}$ BMP-V with or without magnet, $50 \mu \mathrm{g}, 100 \mu \mathrm{g}$ DNA vaccine). The same treatments were repeated on days 8 and 12 . Tumour volumes were monitored for 25 days until the control mice began to die. Data are presented as mean tumour volumes \pm s.d. of five mice per group in a representative experiment. *Indicates $\mathrm{P}<0.05$, all treatments compared with PBS.

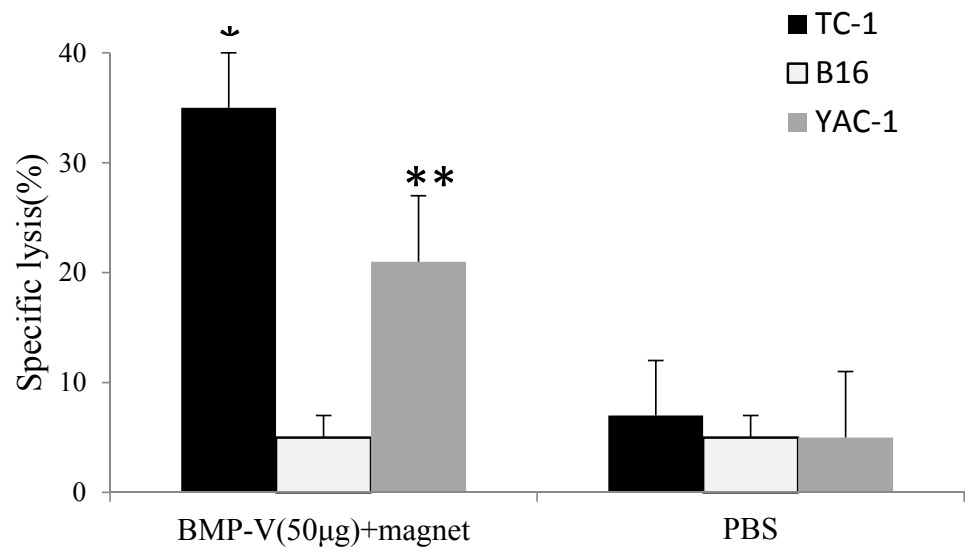

Figure 2: CTL responses in mice $(n=3)$ immunized with $50 \mu \mathrm{g}$ of BMP-V plus magnet for 10 min or PBS on days 4, 8 and 12 after TC-1 cell challenge (day 0). On day 18, pooled spleen cells were harvested, and a standard LDH 
assay was performed. Effector cells from each group were harvested and cytotoxicity was tested against B16, TC-1 YAC-1 tcells at an E:T ratio of 60:1. ***Indicates $P<0.05$, compared with the PBS group.

\section{Discussion}

Bacterial magnetic particles have attracted lots of attention from 1975 [8]. Hopkin demonstrated that BMPs could be used as carrier of drugs to target tumour therapy [9]. The formation of BMPs-PEI-DNA complexes has been illustrated previously Hergt et al. [6] showed that the magnetic character of BMPs as potential therapeutic tools [10].

Compared to other delivery methods, we demonstrated that i.m. injection BMP-carrier gene vaccine plus a magnetic field resulted in better inhibited tumour inhibition. These results showed that a magnetic force may speed up the accumulation of BMP-V on the surface of cells. The magnetic force also strengthened nuclear uptake of magnetic particles in the process of magnetofection. With the help of magnetic force, BMP-V may arrive the surface of the cells more quickly and promote the entry of plasmid DNA into the cells more efficiently.

The small size and favourable dispersion characteristics of BMPs were used in the previous studies [11], but we used the paramagnetism of BMPs to make BMP-DNA vaccine complex. With the help of a magnetic field, we showed that the delivery of the BMP-V could generate greater levels of the E7-specific cytotoxic CD8+ T and induce a specific anti-tumour immune response.

In our study, BMPs carried the gene vaccine (pSLC-E7-Fc) into skin cells, wherein the SLC-E7-Fc fusion protein is subsequently expressed and secreted. The secreted SLC-E7-Fc can recruit a large number of dendritic cells, especially Langerhans cells, at the vaccination site, which may facilitate antigen presentation [12]. This advantage is similar to the gene gun system [13]. However, the mechanism regarding the effects of magnetic field in magnetofection are still unknown and require extensive research for further validation.

DNA vaccine technologies have attracted the attention to develop the efficient way of gene delivery in vivo. BMPs are synthesized in the organism and no toxicity to cells. The cytoplasmic membrane of BMPs provides a presumed good biocompatibility in vivo. In this study, we showed that the BMP vaccine system induce specific immunity. In conclusion, BMPs could be used as a DNA delivery vehicle to enhance anti-tumor effect.

\section{Acknowledgements}

This work was supported by a grant from National Key R\&D Program of China (2017YFC0909900), National Natural Science Foundation of China (No 81501818 of Yishu Tang) and the National Key Clinical Specialties Construction Program of China.

\section{Conflict of Interest}

The authors declare no conflicts of interest. 


\section{References}

1. Bansal A, Jackson B, West K, et al. Multifunctional T-cell characteristics induced by a polyvalent DNA prime/protein boost human immunodeficiency virus type 1 vaccine regimen given to healthy adults are dependent on the route and dose of administration. J Virol 8 (2008): 6458-6469.

2. Donnelly J, Berry K, Ulmer JB. Technical and regulatory hurdles for DNA vaccines. Int J Parasitol 33 (2003): 457-467.

3. Nicolas JF, Guy B. Intradermal, epidermal and transcutaneous vaccination: from immunology to clinical practice. Expert Rev Vaccines 7 (2008): 1201-1214.

4. Bazylinski DA, Frankel RB. Magnetosome formation in prokaryotes. Nat Rev Microbiol 2 (2004): $217-$ 230.

5. Xie J, Chen K, Chen X. Production, modification and bio-applications of magnetic nanoparticles gestated by magnetotactic bacteria. Nano Res 2 (2009): 261-278.

6. Xiang L, Bin W, Huali J, et al. Bacterial magnetic particles (BMPs)-PEI as a novel and efficient non-viral gene delivery system. J Gene Med 9 (2007): 679-690.

7. Liu R, Zhou C, Wang D, et al. Enhancement of DNA vaccine potency by sandwiching antigen-coding gene between secondary lymphoid tissue chemokine (SLC) and IgG Fc fragment genes. Cancer Biol Ther 5 (2006): 427-434.

8. Matsunaga T. Applications of bacterial magnets. Trends Biotechnol 9 (1991): 91-95.

9. Hopkin M. Magnet-making bacteria could target tumours. Nature (2004).

10. Hergt R, Hiergeist R, Zeiberger M. Magnetic properties of bacterial magnetosomes as potential diagnostic and therapeutic tools. J Magnet Magnet Mater 293 (2005): 80-86.

11. Han L, Zhang A, Wang H, et al. Tat-BMPs-PAMAM conjugates enhance therapeutic effect of small interference RNA on U251 glioma cells in vitro and in vivo. Hum Gene Ther 21 (2010): 417-426.

12. Nagira M, Imai T, Hieshima $\mathrm{K}$, et al. Molecular cloning of a novel human $\mathrm{CC}$ chemokine secondary lymphoid-tissue chemokine that is a potent chemoattractant for lymphocytes and mapped to chromosome. $\mathrm{J}$ Biol Chem 272 (1997): 19518-19524.

13. Stoecklinger A, Grieshuber I, Scheiblhofer S, et al. Epidermal langerhans cells are dispensable for humoral and cellmediated immunity elicited by gene gun immunization. J Immunol 179 (2007): 886-893.

Citation: Yishu Tang, Chunxia Zhou, Wenbo Ma, Dongmei Wang, Shuren Zhang. An Enhanced Anti-Tumor Response by Using Bacterial Magnetosomes Gene Administration Platform. Archives of Clinical and Biomedical Research 2 (2018): 145-150.

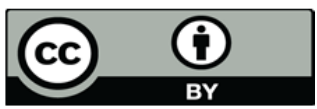

This article is an open access article distributed under the terms and conditions of the Creative Commons Attribution (CC-BY) license 4.0 